\title{
Determinants of polydipsia: VI. Taste of the drinking solution on DRL'
}

EVALYN F. SEGAL AND SAM A. DEADWYLER SAN DIEGO STATE COLLEGE

\begin{abstract}
Abstraet
Three rats on DRL 30 for food developed polydipsia. One drank about the same amount of tap water, saccharine and quinine solutions, but more of saline. One drank about the same amount of water and quinine, but more of saccharine and saline. One drank more saccharine and saline, and less quinine, than water. Drinking in the first two Ss appears to have been controlled by factors additional to taste and thirst, but these appear adequate to explain the third S's drinking.
\end{abstract}

\section{Problem}

Earlier (Segal \& Deadwyler, 1965) we suggested that two of three rats run on a DRL food schedule used water drinking to mediate the spacing of bar presses, while the third $S$ did not. Our inference was based on the facts that drinking followed non-reinforced as well as reinforced bar presses, and followed operation of an empty as well as a full pellet dispenser, in the first two rats, but not in the third. Here we report additional data supporting this inference.

\section{Method}

Part of the experiment was reported in Segal \& Deadwyler (1965). Three adult, male, albino rats were maintained at $80 \%$ of ad lib weight and run daily on a DRL 30 sec. bar-press schedule in a sound-insulated chamber with a bar, a food cup and a water nozzle. Reinforcers were $45 \mathrm{mg}$ Noyes peanut pellets. Sessions lasted for 100 reinforcements.

Following stabilization of bar pressing and drinking, the drinking solution was changed as shown in Fig. 1. Compounds dissolved in a liter of tap water were: S2, 2 grains of saccharine sodium; Q 1.25, 1.25 grains of quinine sulfate; $Q 2.50,2.50$ grains of quinine sulfate; and $\mathrm{SN} 0.3 \%, 3 \mathrm{~g}$ of $\mathrm{NaC} 1$.

\section{Results and Diseussion}

Figure 1 shows the total number of licks and bar presses in the final days of the first water run, and in all succeeding sessions. The horizontal line drawn through the licks data is a visual estimate of the median number of licks over the final days of the first water run, for $\mathrm{A} 2$ and $\mathrm{A} 3$, and the second water run, for $\mathrm{BC} 2$. (The second run for $\mathrm{BC} 2$ appeared to give a more reliable estimate of the S's water-consumption level over the whole experiment.)

Rat A3. Licking was very resistant to change. All points appear to vary about the same baseline, except for a slight overshooting in the third water run. Accompanying every change in solution (including a return to water) there was a more or less temporary disruption of timing (increase in bar presses per 100

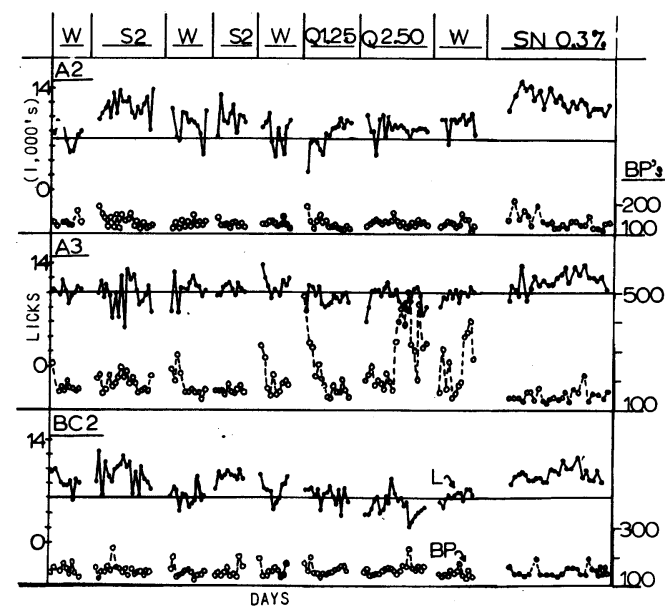

Fig. 1. Total licks in thousands, and total bar presses, of each $\mathbf{S}$, on the final days of the initial water run, and of all succeeding days. Sessions 100 reinforcements long.

reinforcements), even though licking did not change. When the solution was changed to saline A3 finally showed an increase in drinking and bar pressing finally stabilized.

In Fig. 2, the left graph shows that for A3, number of drinks (solid line) varied in an apparently unsystematic manner, and that the mean number of licks per drink (dashed line) was about the same for all solutions except saline, where drinks were slightly longer.

The top right graph shows that except for the slight disruption when quinine was first introduced, changes in solution produced little change in mean interreinforcement time.

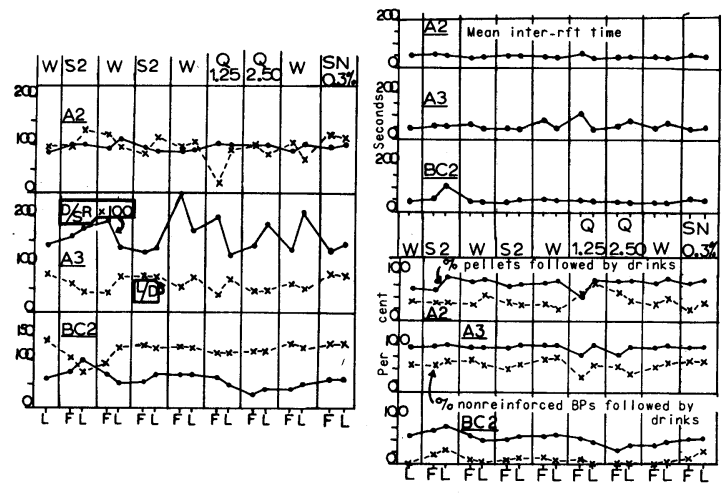

Fig. 2. Left: number and length of drinks. Top right: inter-reinforc ement time averaged over a session. Bottom right: Per cent of reinforced and non-reinforced bar presses followed by drinking. Last day of first water run, first and last day of succeeding runs. 
The lower right graph shows that A3 drank oftener following a reinforced (solid line) than a non-reinforced (dashed line) bar press, but even so, it drank frequently following non-reinforced bar presses. These data were stable for A3 except for the dip in the curves when $Q 1.25$ and $Q 2.50$ were first introduced.

We think A3 used drinking to mediate timing. Because drinking was chained to bar pressing, it did not change much as a function of solution until saline was offered. (Perhaps saline raised consumption by altering the discriminability of the quantity of solution consumed per drink.) The $S$ must have used both quantity consumed and taste cues in mediating bar presses, quantity being implicated by its resistance to change, and taste by its initial effects on timing. $\mathrm{S}$ could control how much it consumed per drink, and did so until offered saline; it could not control taste, and so timing was disrupted when new solutions (other than saline) were offered. (Longer saline drinks offset any disruption due to taste.)

Rat A2. Figure 1 shows that licking rose for saccharine and saline and fell initially on quinine. There was a temporary disruption of timing on initial exposure to each of these solutions, but no disruption on return to plain water. Over the first saccharine run licking did not change but timing recovered. Over the second run, licking did not rise so high, and spacing was not affected. Over the first quinine sessions licking and bar pressing recovered roughly concurrently, and increasing the bitterness of the solution had no further effect.

The left graph of Fig. 2 shows that changes in total number of licks per session were caused by changes in we length, not the number, of drinks. Interreinforcement time was stable. A2, like A3, drank following both reinforced and non-reinforced bar presses, as the bottom right graph shows. The only marked change occurred at $Q 1.25$.

This S, too. appeared to use both taste and quantity consumed as timing cues. Quantity consumed is implicated by the facts that: timing worsened when quantity changed on first exposure to a solution; timing re- covered on continued exposure to the solution, as quantity stabilized; and the recovery of timing on quinine was accompanied by a recovery of drinking. Taste is implicated by the fact that timing remained stable on later exposure to a solution encountered earlier (viz., every water run after the first, and the second saccharine run). This suggests a conditional discrimination, dependent on both quantity and taste: the $\mathrm{S}^{\mathrm{D}}$ for bar pressing was a given quantity of a given taste.

R a t BC2. Results are in marked contrast to the other two Ss. Figure 1 shows little or no disruption of timing on initial or continued exposure to new solutions; and the quantity of solution consumed varied as a simple, direct function of palatability. The left graph of Fig. 2 shows that changes in quantity consumed were caused by changes in number, not length, of drinks (in contrast to A2). Figure 2 also shows that BC2 rarely drank following non-reinforced bar presses, so changes in total number of drinks were caused by changes in percent of pellets followed by drinks.

The facts that BC2 drank less than the other Ss even of saccharine and saline, drank only following eating, drank the same amount whenever it drank, and showed no covariation of drinking and timing, lead us to conclude that BC2 drank because eating pellets made it thirsty, and it drank more or less often of different solutions because they were more or less palatable.

The facts that A2 and A3 drank more of each solution than BC2, drank as much quinine (ultimately) as water, drank following non-reinforced as well as reinforced bar presses, showed disruption of timing when first exposed to a new solution, and recovery of timing as drinking stabilized, lead us to conclude that these Ss drank because eating pellets made them thirsty, and because drinking provided tham with timing cues derived from taste and quantity consumed.

\section{Reference}

Segal, Evalyn F., \& Deadwyler, S. A. Determinants of polydipsia in rats: II. DRL extinction. Psychon. Sci., 1965, 2, 203-204.

Note

1. Supported by NSF GB 1605 and NIMH 8505. 